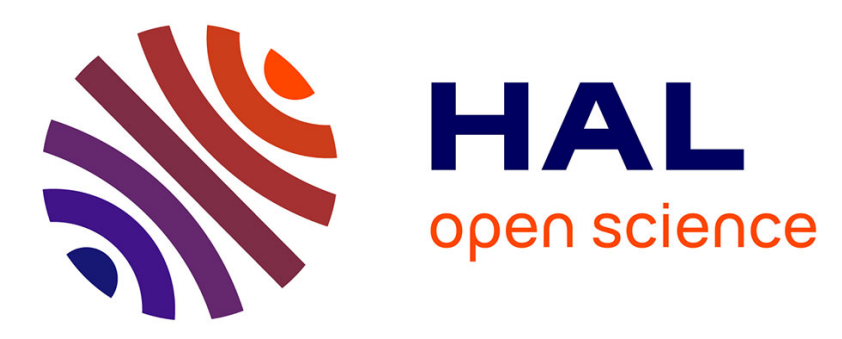

\title{
Perturbation Finite Element Method for Efficient Copper Losses Calculation in Switched Reluctance Machines
}

Moustafa Al Eit, Patrick Dular, Frédéric Bouillault, Claude Marchand, Guillaume Krebs

\section{To cite this version:}

Moustafa Al Eit, Patrick Dular, Frédéric Bouillault, Claude Marchand, Guillaume Krebs. Perturbation Finite Element Method for Efficient Copper Losses Calculation in Switched Reluctance Machines. IEEE Transactions on Magnetics, 2017, 53 (6), pp.7202004. 10.1109/TMAG.2017.2655339 . hal01531370

\section{HAL Id: hal-01531370}

https://hal-centralesupelec.archives-ouvertes.fr/hal-01531370

Submitted on 26 Oct 2020

HAL is a multi-disciplinary open access archive for the deposit and dissemination of scientific research documents, whether they are published or not. The documents may come from teaching and research institutions in France or abroad, or from public or private research centers.
L'archive ouverte pluridisciplinaire HAL, est destinée au dépôt et à la diffusion de documents scientifiques de niveau recherche, publiés ou non, émanant des établissements d'enseignement et de recherche français ou étrangers, des laboratoires publics ou privés. 


\title{
Perturbation Finite Element Method for Efficient Copper Losses Calculation in Switched Reluctance Machines
}

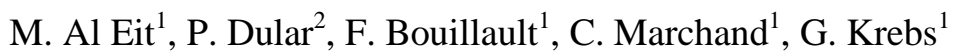 \\ ${ }^{1}$ GeePs, C.N.R.S UMR 8507, CentraleSupélec, Paris-Sud University, Paris-Saclay University, \\ Sorbonne University and Pieree-and-Marie-Curie University, Gif-sur-Yvette 91192, France \\ ${ }^{2}$ Department of Electrical Engineering and Computer Science, University of Liège, Liège 4000, Belgium
}

\begin{abstract}
Copper losses dissipated in the windings of electric machines are the sum of classical ohmic DC losses and additional AC eddy current losses. In fact, the level of eddy current losses is strongly correlated to the manner of disposition of coil conductors in machine slots. Then, to improve the efficiency in electric machines, the selection of an optimal winding configuration becomes substantial. Since eddy current losses derive from the strong electromagnetic coupling between current density and time-dependent magnetic field which cannot be solved easily, numerical analyses such as particularly the one using the finite element method are often used. As for the finite element modeling, it can employ moving band technique to perform the rotor motion and Newton-Raphson iterations to deal with the nonlinear behavior of magnetic circuits. It leads then to a substantial computational time that hinders any process of conception or optimization of winding geometries. To overcome this issue, a 2D finite element model reduction based on the perturbation method is proposed. It starts from one approximate finite element solution of a simplified complete machine modeling to find fast but accurate solutions in slots subdomains when any variation of geometrical or physical data occurs. It allows adapting nonconforming meshes and provides clear advantages in repetitive analyses when we search the optimized winding configuration for a given number of turns.
\end{abstract}

Index Terms - Finite-element analyses, Eddy currents, Perturbation method, Boundary conditions, Switched reluctance machine.

\section{INTRODUCTION}

$\mathrm{T}$ HE eddy current loops in copper conductors exist due to the strong electromagnetic coupling between current density and penetrating time-varying magnetic field[1,2]. By reason of the fact that this interaction between electric and magnetic variables is described mathematically by a complex partial differential equation that cannot be solved easily, finite element (FE) methods are usually used to give a numerical solution. In case of electric machines, FE modeling can integrate moving band technique to perform the rotor motion and Newton-Raphson iterations to take into account the nonlinearity of magnetic circuits. It leads accordingly to a substantial calculation time which hinders any process of conception or optimization. Particularly in this paper, we are interested in the strong influence of the coil geometric disposition in machine slots on the level of eddy current losses dissipated in the windings of switched reluctance machines (SRM). To shorten the calculation time while selecting by means of FE analyses the optimal winding configuration, this paper suggests a model reduction technique which is akin to perturbation $\mathrm{FE}$ method [3,4]. It profits from only one approximate full FE solution based on a uniform current density, a simplified winding and a coarse mesh model to find fast solutions in the slots subdomains when any variation of winding geometrical or physical data occurs. The reduced FE (RFE) model solutions are called perturbations; they are studied under Dirichlet and Neumann boundary conditions (BC) in a test case example to end up with an application on an SRM. In order to validate the reduced problem, the results are compared with a reference complete FE (CFE) model.

\section{FinITE ELEMENT MODELING}

At low frequencies the displacement currents are neglected.
Maxwell's equations concerned then to describe the electromagnetic behavior in a domain $\Omega$ of boundary $\Gamma$ $\left(\Gamma=\Gamma_{1} \cup \Gamma_{2}\right.$ and $\Gamma_{1} \cap \Gamma_{2}=\{\}$ ) (Fig. 1) and the associated constitutive medium relationships are given by:

$$
\begin{gathered}
\nabla \times \vec{H}=\vec{J}, \nabla \cdot \vec{B}=0, \nabla \times \vec{E}=-\partial \vec{B} / \partial t \\
\vec{H}=v(|\vec{B}|) \vec{B}, \vec{J}=\sigma \vec{E}
\end{gathered}
$$

where $\vec{B}$ is the magnetic flux density vector, $\vec{H}$ is the magnetic field vector, $\vec{E}$ is the electric field vector, $\vec{J}$ is the current density vector, $\sigma$ is the conductivity and $v$ is the reluctivity. Reluctivity $v$ may depend on $\vec{B}$ in ferromagnetic materials, i.e., with a nonlinear behavior. To impose the uniqueness of the solution, homogenous Neumann and Dirichlet BC set to zero, can be considered such that:

$$
\vec{H} \times \vec{n}=\overrightarrow{0} \text { on } \Gamma_{2} ; \quad \vec{B} \cdot \vec{n}=0 \text { on } \Gamma_{2}
$$

with $\vec{n}$ the outward boundary unit normal vector.

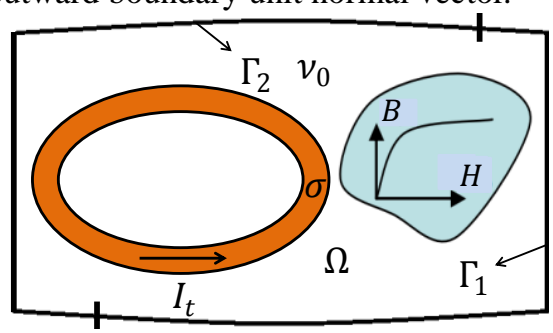

Fig. 1. Nonlinear electromagnetic problem coupled with electric circuits

An easy coupling with electric circuits can be achieved using the magnetic vector potential $\vec{A}$ defined implicitly by $\vec{B}=\nabla \times \vec{A}$ and the electric scalar potential $V$ with $\vec{E}=$ $-\partial \vec{A} / \partial t-\nabla V$. To ensure the unicity of $\vec{A}$, the Coulomb gauge is added. The resulting vector potential formulation from (1) and (2) is given by: 


$$
\nabla \times(v(|\nabla \times \vec{A}|) \nabla \times \vec{A})=-\sigma \partial \vec{A} / \partial t-\sigma \nabla V
$$

A combined circuit equation reflects that, at the instant $t$, the current feeding the conductor of cross section $S$ is $I_{t}$ :

$$
I_{t}=\iint_{S} \vec{J} \cdot \overrightarrow{d s}=\iint_{S}(-\sigma \partial \vec{A} / \partial t-\sigma \nabla V) \cdot \overrightarrow{d s}
$$

The 2D FE formulation of (4), together with the electric circuit equation (5) and the backward Euler scheme for time discretization, lead to the following nonlinear matrix system [1,5]:

$$
\left(\begin{array}{cc}
{\left[S\left(A_{t}\right)\right]+\frac{[T]}{\Delta t}} & {[D]} \\
{[D]^{t}} & \Delta t[G]
\end{array}\right)\left[\begin{array}{c}
{\left[A_{t}\right]} \\
{\left[\Delta V_{t}\right]}
\end{array}\right]=\left[\begin{array}{c}
\frac{[T]}{\Delta t}\left[A_{t}\right] \\
\Delta t\left[I_{t}\right]+[D]^{t}\left[A_{t-\Delta t}\right]
\end{array}\right]
$$

At the instant $t, \Delta V_{t}$ is the electric potential drop (voltage) per unit length through an individual conductor. With $\mathrm{N}_{\mathrm{c}}$ conductors $\left(\mathrm{N}_{\mathrm{c}}>1\right)$, the size of the vectors $\left[\Delta V_{t}\right]$ and $\left[I_{t}\right]$ is $\mathrm{N}_{\mathrm{c}} \times 1 . \Delta \mathrm{t}$ represents the time step and $\left[A_{t}\right]$ is the vector of unknown nodal magnetic vector potentials at the instant $t$. It is of size $N_{n} \times 1$ where $N_{n}$ is the total number of mesh nodes without the prescribed ones with Dirichlet BC. The NewtonRaphson method is employed to consider the iron nonlinear magnetic behavior and the moving band technique [6] is used to perform the rotor motion in case of rotating machines.

\section{Perturbation Model Reduction Method}

\section{A. Position of the problem}

The copper losses in electric machines are subdivided into classical ohmic DC losses and additional eddy current losses occurring due to the displacement of the current. The displacement of the current is manifested by the non-uniform current density distribution along the conductor cross section.

In fact, the eddy current losses dissipated in the winding of electric machines take place due to three different current displacement effects; we distinguish the skin effect, the proximity effect and the effects due to the rotor motion and the magnetic circuit saturation. When the skin effect is caused by the time-varying magnetic field of the conductor current itself, the proximity effect is caused by external time-varying magnetic fields sent by adjacent conductors' currents. Furthermore, the motion in case of salient rotor machines causes a significant deviation of the flux lines throughout the slot. The magnetic circuit saturation, around the slot subdomains, can be the origin of additional stray flux lines than can alter the magnetic flux distribution across the slot conductors. These dual additional effects due to rotor motion and saturation phenomena force hence a strong twodimensional magnetic flux throughout the slot which leads therefore to additional eddy current losses. The disposition of the coil conductors in the slots with respect to magnetic flux lines is deterministic then in terms of the dissipated amount of additional eddy current losses.

Based therefore on the assumption that the amount of eddy current losses is strongly correlated to the manner of disposition of coil conductors in machine slots, it is firmly recommended then to select the optimal winding configuration in terms of copper losses. In the particular case of SRM, the amount of eddy currents can be significant since it features a double salient geometry and is generally equipped with a concentrated winding that increases the likelihood of localized saturation in the stator poles. To insure a better accuracy in the computation of copper losses in electric machines, FE analysis has to take into account the rotor motion and the non-linearity of magnetic circuits. When the CFE modeling treats the rotor motion and the non-linearity of the magnetic circuits by means of the moving band technique and the tedious NewtonRaphson iterations, it leads to a substantial computational time and a relatively large storage capacity that preclude any kind of conception or optimization processes.

\section{B. Model reduction using the perturbation method}

To select the optimal winding configuration in terms of copper losses, while avoiding for each winding configuration a CFE model of the machine, perturbation FE method is used. Only one full FE model is considered where a coarse mesh, a simplified one-turn coil and a uniform current density distribution are employed. Once it is solved, we pick up the information on the slot boundary, from which either the vector potential or its normal derivative are applied as Dirichlet or Neumann BC respectively to the slot subdomains where we integrate the real investigated winding configurations of conductivity $\sigma$. Accordingly, the proposed model reduction consists in solving the problem only in the slots' subdomains where the solutions are called perturbations $[3,4]$.

In fact, the Dirichlet BC imposes the normal component of the magnetic field on the slots boundaries and acts then on behalf of the magnetic circuit and the salient rotor motion influences on the circulation of the magnetic flux lines. In the particular case of Litz and twisted wire conductors which try to homogenize the current density distribution, the application of a pure Dirichlet fits very well [1]. This seems obvious since the simplified start up problem is solved under a uniform current density. Nevertheless, in case of solid conductors the flux lines displacements due to eddy currents are extensive. Hence, the significant variation of the normal component of the magnetic field must then be taken into account by an appropriate BC. In fact, since the aim of Neumann BC is to impose the tangential component of the magnetic field, it allows simultaneously correcting the normal component and appears to be the appropriate $\mathrm{BC}$ that takes into account the significant flux lines displacement in case of solid conductors. The effect of the $\mathrm{BC}$ on the reduced model perturbations is studied in the next sub-section on a test-case example.

\section{Boundary conditions effects}

To study the effect of BC on the perturbation FE solution in the reduced problem, a solid conductor located in a simplified laminated slot is considered (Fig. 2). The conductor is fed, at the frequency of $300 \mathrm{~Hz}$, by a rectified sinusoidal current close to that of SRM. To apply the perturbation method, an approximate problem considering a uniform current density and a simplified coarse mesh model is first solved. It gives the $\mathrm{BC}$ for the RFE perturbation problem consisting solely of the 
slot subdomain. Once it is solved, we pick up the information on the boundary $\Gamma_{\mathrm{c}}\left(\Gamma_{\mathrm{c}}=\Gamma_{\mathrm{cD}} \cup \Gamma_{\mathrm{cN}}\right.$ and $\left.\Gamma_{\mathrm{cD}} \cap \Gamma_{\mathrm{cN}}=\{\}\right)$, from which either the vector potential or its normal derivative are applied as Dirichlet or Neumann BC respectively to the slot reduced domain $\Omega_{r}$ of boundary $\Gamma_{\mathrm{r}}\left(\Gamma_{\mathrm{r}}=\Gamma_{\mathrm{rD}} \cup \Gamma_{\mathrm{rN}}\right.$ and $\Gamma_{\mathrm{rD}} \cap \Gamma_{\mathrm{rN}}=\{\}$ ) (Fig. 2). In the reduced problem, the solid conductor cross section of conductivity $\sigma$ is governed by the circulation of additional eddy currents since the current density is given now by $\vec{J}=-\sigma \partial \vec{A} / \partial t-\sigma \overrightarrow{\operatorname{grad}}(V)$. Usually in the reduced problem, in order to insure a better accuracy in the calculation of eddy currents, a finer mesh is adopted. The mortar element method can therefore be used to adapt nonconforming meshes [7]. However, in our test-case example, to avoid any kind of errors that can occurs due to the adaptation of nonconforming meshes and for a better study of the effect of the BC on the flux lines circulation, the same mesh density is used. The nonconforming meshes will be taken into account in the case of the SRM in the next section.

Two different reduced problems are studied. By analogy to [1], in the first reduced problem, the vector potential solution is captured from the entire boundary $\Gamma_{\mathrm{c}}$ from the approximate model and applied as a pure Dirichlet BC on the reduced problem boundary $\Gamma_{\mathrm{r}}$. This leads to a significant error of $63 \%$ on the calculation of the copper losses dissipated in the solid conductor (Fig. 3). This result is clarified by the inconsistency of the distribution of magnetic flux lines and current density in the slot domain and the conductor cross section respectively compared to that of a reference CFE model (Figs. 4 and 5).

It should be noted, seeing Fig. 4, that in the approximate CFE model with uniform current density, the flux lines are almost perpendicular to the boundary $\Gamma_{\mathrm{cN}}$ which is normal since $\Gamma_{\mathrm{cN}}$ is the common boundary with the laminated magnetic circuit; the flux lines mostly penetrate perpendicularly the magnetic circuit. However, these flux lines are almost parallel to the upper boundary $\Gamma_{\mathrm{cD}}$. In the reduced problem, if the eddy current loops occur, they lead to a significant displacement of the current toward the upper boundary (Fig. 5) which leads simultaneously to a significant deviation of the flux lines as shown in Fig. 4. The most altered magnetic field component on $\Gamma_{\mathrm{rN}}$ will therefore be the normal component to the boundary, while that of the boundary $\Gamma_{\mathrm{rD}}$ will be the tangential component. The Dirichlet BC imposes the normal component and enables to correct the tangential one, whereas the Neumann BC imposes the tangential component and allows to correct the normal one. A Dirichlet $\mathrm{BC}$ and a Neumann $\mathrm{BC}$ should be imposed on $\Gamma_{\mathrm{rD}}$ and $\Gamma_{\mathrm{rN}}$ respectively so that the flux lines deviations due to the circulation of eddy currents should be taken into account. The second reduced problem is then solved under mixed Neumann-Dirichlet BC where the quality of the solution is highly improved. This fact can be clearly shown on the current density and the flux lines distributions which correspond with a good precision to the results of a reference CFE model solved with a current density including the eddy currents throughout the conductor cross section (Figs. 4 and 5). Consequently, the total copper losses curve in the reduced problem matches very well that of the CFE model with an error that does not exceed $0.01 \%$ (Fig. 3).

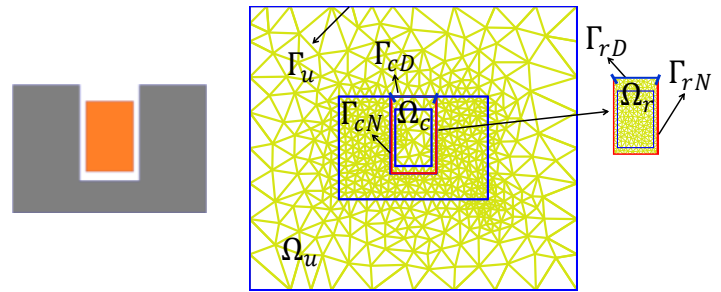

Fig. 2. Test-case example: solid conductor located in a lamination slot.

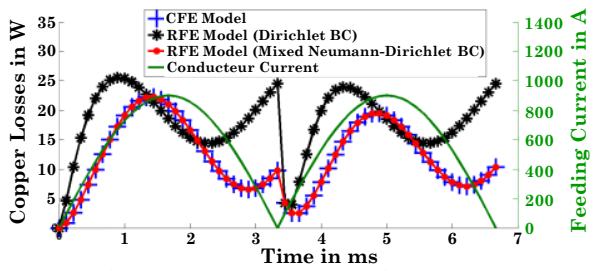

Fig. 3. Evolution of copper losses obtained from the CFE and RFE models

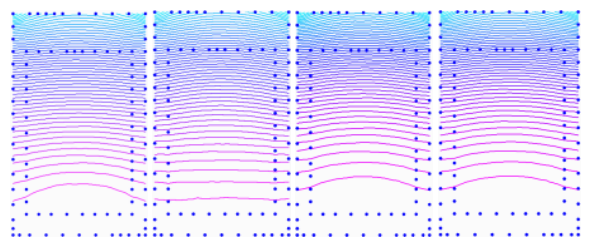

Fig. 4. Distribution of the magnetic flux lines across the lamination slot. Approximate FE model with uniform current density (left), RFE model under Dirichlet BC (middle-left), RFE model under Mixed Neumann-Dirichlet BC (middle-right), reference CFE model (right). (Time $=4.7 \mathrm{~ms}$ ).

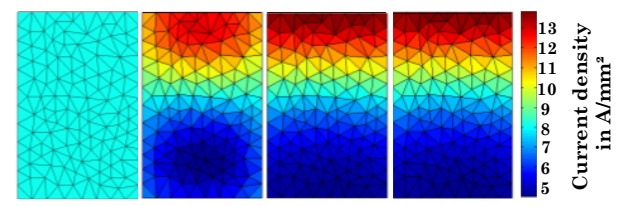

Fig. 5. Current density distribution in the conductor cross section. Approximate FE model with uniform current density (left), RFE model under Dirichlet BC (middle-left), RFE model under Mixed Neumann-Dirichlet BC (middle-right), reference CFE model (right). (Time $=4.7 \mathrm{~ms}$ ).

\section{APPLICATION EXAMPLE AND RESUltS}

An SRM 8/6 is studied. As shown in Fig. 6, half of the machine is modeled for periodic reasons and only one phase is fed. It consists of two coils connected in series; each of them has 18 turns. These turns can be distributed in machine slots in different ways which leads to various winding configurations (Fig. 7). It should be noted that $(\mathrm{m} \times \mathrm{n})$ configuration means that we have $\mathrm{m}$ horizontal layers with $\mathrm{n}$ conductors by layer. In the particular case of SRM, since back and forth conductors of the same coil are subject to different magnetic stray fields (Fig. 6), their slot subdomains are studied separately (Fig. 7). Besides the flux lines penetrating the magnetic circuit in the normal direction with respect to the slot edges, the flux lines in the air gap region try to join the moving rotor. They penetrate therefore the slot boundary common with the air gap also in the normal direction. Since the normal component of the magnetic field is the one to be corrected in the reduced problem, the variation of the magnetic vector potential with respect to the normal direction is collected from the boundaries $\Gamma_{\mathrm{c} 1}$ and $\Gamma_{\mathrm{c} 2}$ of the slots domains $\Omega_{\mathrm{c} 1}$ and $\Omega_{\mathrm{c} 2}$ in the 
approximate FE model first solved (Fig. 6) and is applied as Neumann $\mathrm{BC}$ on $\Gamma_{\mathrm{r} 1}$ and $\Gamma_{\mathrm{r} 2}$ of the reduced problem domains $\Omega_{\mathrm{r} 1}$ and $\Omega_{\mathrm{r} 2}$ (Fig. 7). For a well-posed problem for the reduced model, we must impose a Dirichlet $\mathrm{BC}$ on a single node of the boundary. It is the value of the magnetic vector potential calculated in the first solved approximate FE model. We therefore insure that the solution of the reduced problem integrating the studied winding configuration is a kind of correction of the solution in the approximate full model solved with the uniform current density distribution.
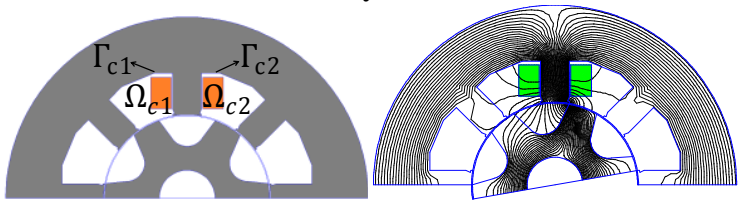

Fig. 6. Approximate model with a simplified 1-turn winding (left). Nonsymmetrical flux lines distribution in back and forth conductors' slots (right).
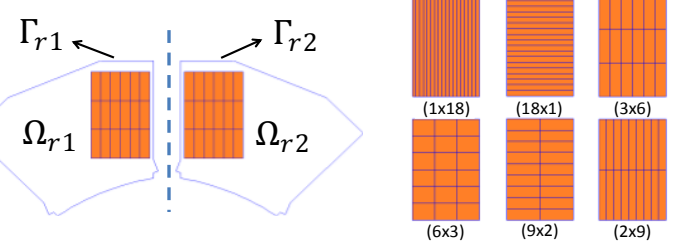

Fig. 7. RFE models (left). The six different winding configurations (right).

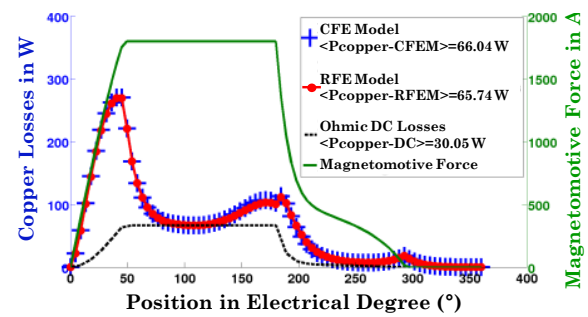

Fig. 8. Copper losses calculated in the left coil side conductors.

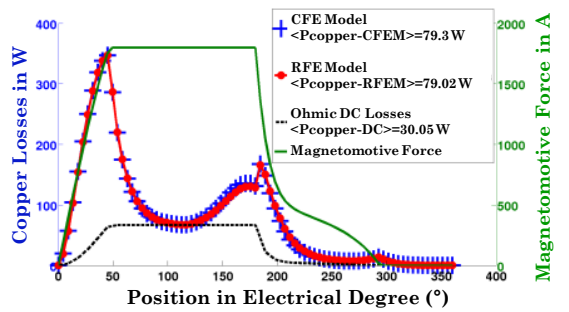

Fig. 9. Copper losses calculated in the right coil side conductors.

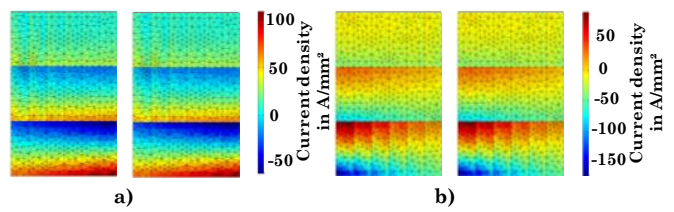

Fig. 10. a) Current density distribution in the left coil side conductors. CFE model (right), RFE model (left). b) Current density distribution in the right coil side conductors. CFE model (right), RFE model (left). (Rotor position $\left.\theta=48^{\circ}\right)$.

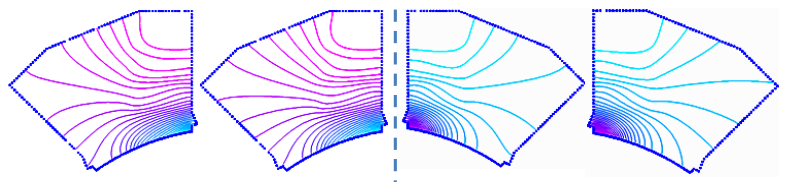

a)

b)

Fig. 11. a) Flux lines repartition throughout the left slot domain. CFE model (right), RFE model (left). b) Flux lines repartition throughout the right slot domain. CFE model (right), RFE model (left). (Rotor position $\theta=48^{\circ}$ ).

We remark that the model reduction solution matches very well the one of the reference CFE model. The results are in agreement not only with respect to the global quantities (Figs. 8 and 9) but also regarding the local one (Figs. 10 and 11). It should be noted that the current density distributions and the flux lines repartitions are represented in Figures 10 and 11 respectively at the rotor position of $\theta=48$ electrical degrees where the copper losses represent their maximal values. It is clear that the losses are higher and the extension of the current density values is more important in the right coil side conductors; these are the conductors that encounter the closest rotor pole in their proximity during the magnetization i.e. the current rise phase. The mean values of copper losses in the left and the right coils conductors are calculated in the different winding configurations (Table 1). By means of the RFE model that shows a speed up factor close to 15 and an error $\varepsilon_{\mathrm{m}}$ that does not exceed $2 \%$ compared to the CFE model, we can concretely select the optimal configuration which is represented by the horizontal conductors' disposition $(18 \mathrm{x} 1)$.

TABLE I

MEAN COPPER LOSSES IN THE COIL'S RIGHT AND LEFT SIDES

\begin{tabular}{ccccccc}
\hline \hline & \multicolumn{5}{c}{ Mean values of copper losses (W) } \\
\cline { 2 - 7 } Configuration & \multicolumn{3}{c}{ Coil's left side } & \multicolumn{3}{c}{ Coil's right side } \\
\cline { 2 - 7 } & $\begin{array}{c}\text { CFE } \\
\text { model }\end{array}$ & $\begin{array}{c}\text { RFE } \\
\text { model }\end{array}$ & $\boldsymbol{\varepsilon}_{\boldsymbol{m}}(\%)$ & $\begin{array}{c}\text { CFE } \\
\text { model }\end{array}$ & $\begin{array}{c}\text { RFE } \\
\text { model }\end{array}$ & $\boldsymbol{\varepsilon}_{\boldsymbol{m}}(\%)$ \\
\hline $\mathbf{( 1 x 1 8 )}$ & 61.26 & 60.54 & 1.17 & 76.72 & 74.49 & 1.61 \\
\hline $\mathbf{( 2 x 9 )}$ & 72.53 & 71.95 & 0.86 & 89.65 & 88.90 & 0.83 \\
\hline $\mathbf{( 3 x 6 )}$ & 66.05 & 64.74 & 0.46 & 79.30 & 79.02 & 0.35 \\
\hline $\mathbf{( 6 x 3 )}$ & 47.76 & 47.63 & 0.26 & 54.54 & 54.44 & 0.19 \\
\hline $\mathbf{( 9 x 2 )}$ & 42.17 & 42.00 & 0.39 & 50.59 & 50.41 & 0.35 \\
\hline $\mathbf{( 1 8 x 1 )}$ & 37.80 & 38.03 & 0.59 & 54.39 & 53.78 & 1.11 \\
\hline \hline
\end{tabular}

\section{CONCLUSION}

The presented 2D model reduction based on the perturbation method allows accurate and fast calculation of machine copper losses. It shows clear interest in repetitive analysis such as winding optimization processes.

\section{REFERENCES}

[1] M. Al Eit, F. Bouillault, C. Marchand and G. Krebs, "2-D Reduced Model for Eddy Currents Calculation in Litz Wire and Its Application for Switched Reluctance Machine," in IEEE Transactions on Magnetics, vol. 52, no. 3, pp. 1-4, March 2016.

[2] M. Klauz and D. G. Dorrell, "Eddy Current Effects in a Switched Reluctance Motor," in IEEE Transactions on Magnetics, vol. 42, no. 10, pp. 3437-3439, Oct. 2006.

[3] R. V. Sabariego and P. Dular, "A Perturbation Approach for the Modeling of Eddy Current Nondestructive Testing Problems With Differential Probes," in IEEE Transactions on Magnetics, vol. 43, no. 4, pp. 1289-1292, April 2007.

[4] P. Dular, R. V. Sabariego, M. V. Ferreira da Luz, P. Kuo-Peng and L. Krahenbuhl, "Perturbation Finite Element Method for Magnetic Model Refinement of Air Gaps and Leakage Fluxes," in IEEE Transactions on Magnetics, vol. 45, no. 3, pp. 1400-1403, March 2009.

[5] P. Lombard and G. Meunier, "A general purpose method for electric and magnetic combined problems for 2D, axisymmetric and transient systems," in IEEE Transactions on Magnetics, vol. 29, no. 2, pp. 17371740, Mar 1993.

[6] B. Davat, Z. Ren and M. Lajoie-Mazenc, "The movement in field modeling," in IEEE Transactions on Magnetics, vol. 21, no. 6, pp. 22962298, Nov 1985.

[7] O. J. Antunes, J.P.A. Bastos, N. Sadowski, A. Razek, L. Santandrea, F. Bouillault, F. Rapetti, "Comparison between nonconforming movement methods," in IEEE Transactions on Magnetics, vol. 42, no. 4, pp. 599602, April 2006. 